\title{
FLEXIBEAM: ANALYTIC SPATIAL FILTERING BY BEAMFORMING
}

\author{
Paul Hurley *, Matthieu Simeoni ${ }^{*, \dagger}$ \\ * IBM Zurich Research Laboratory, CH-8803 Rüschlikon, Switzerland \\ † École Polytechnique Fédérale de Lausanne (EPFL), CH-1015 Lausanne, Switzerland
}

\begin{abstract}
We propose a new, general, method for spatial filtering by beamforming. The desired filter, specified analytically on an $n$-dimensional sphere, is extended to $n+1$-dimensional Euclidean space. A continuous beamforming function is then obtained by the $n+1$ dimensional Fourier transform of the extended filter. The beamforming weight at a given array element corresponds then to a sample of the function at the array element location.

The scheme is a generalisation of focused beamforming on a single point by phase difference alignment. The analytic framework allows tractable, stable determination of beamforming weights, and for clear filter specification. By avoiding approximating a Dirac, desired areas can be covered with reduced side lobes. Multiple areas may be targeted simultaneously. In communications applications, channel information updates can be reduced, and movement accounted for. A WiFi demonstration shows that more flexible beam-shapes can be beneficial for real-life examples, factoring in attenuation.
\end{abstract}

Index Terms - Beamforming, array signal processing, mobile communications, spatial filtering

\section{INTRODUCTION}

Most array beamforming is of the focused variety: have a single point in mind, and steer by phase difference alignment to get there as best you can [1 2]. While this minimises noise in and around the target, there are drawbacks. A Dirac is a difficult (impossible) thing to approximate, and significant signal sneaks in from side lobes. It is also particularly sensitive. Small errors in direction of interest estimates can have consequences.

Devices, and the people carrying them, often move, and constant channel updates are required to account for this. For example, 802.11ac WiFi requires a single device to relay its channel state every $0.1 \mathrm{~s}$. For MU-MIMO, beamforming to multiple users at the same time requires communication on the order of $0.01 \mathrm{~s}[3]$.

And ultimately, many applications want to target around general areas of interest. A radio astronomer often wants a portion of the sky. An ultrasound picture is a scan of a region. Mobile phone mast designers want to concentrate energy where most devices are.

In the paper we outline a technique we call flexibeam for determining beamforming weights that, when applied, approximate a given spatial filter design. A key concept is that of a beamforming function $w(\boldsymbol{p})$ defined over Euclidean space (the examples shown are either 2 or 3D). Weights are then samples of this function at array element positions. An antenna located at position $\boldsymbol{p}$ will have weight $w(\boldsymbol{p})$ (for unit wavelength).

To arrive at this function, we take the spatial filter specified over the sphere $\mathbb{S}^{n}$ (e.g., the circle $\mathbb{S}^{1}$ or sphere $\mathbb{S}^{2}$ ), and extend it to $n+1$ dimensional Euclidean space. The beamforming function is then defined as the $n+1$ dimensional Fourier transform of the extended filter.

The scheme is a generalisation of focused beamforming, and far more flexible (hence the name). The analytic framework turns out to be a natural extension that allows tractable, numerically stable determination of beamforming weights, and for clear specification of the desired filter.

Aiming for areas rather than points minimises the required update rate, and reduces the communication requirement. Arbitrary distributions of devices can be targeted at once. Side lobes and power requirements can be reduced substantially. In MIMO systems, the optimisation tradeoff between (focused) beamforming and spatial diversity (multiple replicas of the radio signal from different directions) [4] can be circumvented.

We use WiFi transmission as an illustration for flexibeam. From a specified preference radiation pattern, a spatial filter and its extension are obtained, compensation for attenuation performed, and the beamforming derived.

\subsection{Relation to prior work}

Matched beamforming ${ }^{1}$ is the most extensively used method in practise. A vast collection of data-dependent beamformers seek to optimise certain features of the incoming signals [2, 6]. They usually offer better performance than data-independent techniques, but are computationally expensive and need to be calculated on-the-fly. For example, the popular Minimum Variance Directionless Response (MVDR) [7] beamformer seeks to maximise signal power from a given direction and to suppress other contributions.

The idea to spatially filter using beamforming is not new [2]. However, despite the apparent versatility of the technique, existing beamforming strategies suffer from a lack of flexibility, and do not offer a way to approximate arbitrary filters. Some are restrictive in the array layout - for example, beamforming with linearly and equally spaced or planar and factorable arrays is a polynomial approximation problem, and a range of filters can be achieved with the Dolph-Chebyshev tapering method [2]. Others involve an illposed optimisation, that often result in weights with very large moduli, and hence unreliable beam-shapes and noise magnification [2]. For spherical arrays, bandwidth limited filters can be achieved by beamforming in the spherical domain [6].

Collaborative randomised beamforming [8] seeks to maximise information, after correlation, of beams coming from different collections of antennas. The result is a wider field of view than matched beamforming. Combining flexibeam in a collaborative fashion could also be something useful.

\footnotetext{
${ }^{1}$ Known by many names such as delay and sum [2 1], phase and delay, conjugate beamforming, beam steering/focusing [5] etc.
} promotional purposes or for creating new collective works for resale or redistribution to servers or lists, or to reuse any copyrighted component of this work in other works, must be obtained from the IEEE. Contact: Manager, Copyrights and Permissions / IEEE Service Center / 445 Hoes Lane / P.O. Box 1331 / Piscataway, NJ 08855-1331, USA. Telephone: + Intl. 908-562-3966. DOI: 10.1109/ICASSP.2016.7472203 


\section{FLEXIBEAM}

\subsection{Background}

Beam-shapes designed using flexibeam can be used to receive or transmit. For simplicity, we explain the receiving case. Consider an array of $L$ omni-directional receiving antennas, with unit gains and positions $\boldsymbol{p}_{1}, \ldots, \boldsymbol{p}_{L} \in \mathbb{R}^{n}$. A beamformed signal $y(t)$ is formed by combining linearly each antenna's signal $x_{i}(t) \in \mathbb{C}$ using complex weights $w_{i}$ :

$$
y(t)=\sum_{i=1}^{L} w_{i}^{*} x_{i}(t) .
$$

Assume emitting sources are in the far field, and hence can be seen as lying on the unit sphere $\mathbb{S}^{n-1}$. Restrict also emitted source signals to narrow-band. Without loss of generality, let the wavelength of the incoming signals be 1 . Hence, the signal at the $i$ th antenna, originating from direction $\boldsymbol{r} \in \mathbb{S}^{n-1}$ admits the so-called baseband representation and can be written,

$$
x_{i}(t)=\int_{\mathbb{S}^{n-1}} s(t, \boldsymbol{r}) e^{-j 2 \pi\left\langle\boldsymbol{r}, \boldsymbol{p}_{i}\right\rangle} d \Omega+n_{i}(t),
$$

where $\boldsymbol{p}_{0} \in \mathbb{R}^{n}$ is the chosen phase reference centre, and $n_{i}(t)$ is complex additive noise. Plugging (2) into (1), yields:

$$
y(t)=\int_{\mathbb{S}^{n-1}} s(t, \boldsymbol{r}) b^{*}(\boldsymbol{r}) d \Omega+\sum_{i=1}^{L} w_{i}^{*} n_{i}(t),
$$

where $b(\boldsymbol{r})=\sum_{i=1}^{L} w_{i} e^{j 2 \pi\left\langle\boldsymbol{r}, \boldsymbol{p}_{i}\right\rangle}$ is the array beam-shape. Beamforming is effectively spatial filtering the incoming signals by the beam-shape $b^{*}(\boldsymbol{r})$.

\subsection{Beamforming defined over a field}

Flexibeam's main departure from the state-of-the-art is to consider a notional continuous field of antennas covering $\mathbb{R}^{n}$. Over this field is a sensor function $x(t, \boldsymbol{p}) \in \mathcal{L}^{2}\left(\mathbb{R}^{n}, \mathbb{C}\right)$, which describes the signal observable at position $\boldsymbol{p}$ and time $t$, and extends (2) to cover all points in $\mathbb{R}^{n}$ :

$$
x(t, \boldsymbol{p})=\int_{\mathbb{S}^{n-1}} s(t, \boldsymbol{r}) e^{-j 2 \pi\langle\boldsymbol{r}, \boldsymbol{p}\rangle} d \Omega+n(t, \boldsymbol{p}),
$$

where $n(t, \boldsymbol{p}) \in \mathcal{L}^{2}\left(\mathbb{R}^{n}, \mathbb{C}\right)$ is the noise observable at position $\boldsymbol{p}$ and time $t$. The concept of beamforming weight can also be extended to all $\boldsymbol{p} \in \mathbb{R}^{n}$ by a beamforming function $w \in \mathcal{L}^{2}\left(\mathbb{R}^{n}, \mathbb{C}\right)$, and beamforming becomes a continuous inner-product between the beamforming and sensor functions:

$$
\begin{aligned}
y(t) & =\langle w(\boldsymbol{p}), x(t, \boldsymbol{p})\rangle_{\mathcal{L}^{2}\left(\mathbb{R}^{n}, \mathbb{C}\right)}=\int_{\mathbb{R}^{n}} w^{*}(\boldsymbol{p}) x(t, \boldsymbol{p}) d \boldsymbol{p} \\
& =\int_{\mathbb{R}^{n}} w^{*}(\boldsymbol{p})\left(\int_{\mathbb{S}^{n-1}} s(t, \boldsymbol{r}) e^{-j 2 \pi\langle\boldsymbol{r}, \boldsymbol{p}\rangle} d \Omega+n(t, \boldsymbol{p})\right) d \boldsymbol{p} \\
& =\int_{\mathbb{S}^{n-1}} s(t, \boldsymbol{r}) \hat{w}^{*}(\boldsymbol{r}) d \Omega+n_{b}(t) .
\end{aligned}
$$

The beam-shape for the notional antenna field is then $\hat{w}(\boldsymbol{r}) \in$ $\mathcal{L}^{2}\left(\mathbb{S}^{n-1}, \mathbb{C}\right)$. It re-weights the signals coming from various directions differently, and hence defines a spatial filter, which is linked to the beamforming function through

$$
\hat{w}(\boldsymbol{r})=\int_{\mathbb{R}^{n}} w(\boldsymbol{p}) e^{j 2 \pi\langle\boldsymbol{r}, \boldsymbol{p}\rangle} d \boldsymbol{p} .
$$

So far the beamforming function has been only defined over $\mathbb{S}^{n-1}$. We thus propose to extend it to $\mathbb{R}^{n}$ to induce an $n$-dimensional Fourier transform relationship. Let then $\hat{\omega}: \mathbb{R}^{n} \rightarrow \mathbb{C}$ be a function whose $n \mathrm{D}$ Fourier transform exists, and on the hypersphere $\mathbb{S}^{n-1}$ is equal to the target filter we would like to achieve. We call $\hat{\omega}(\boldsymbol{r})$ thus designed the extended filter.

Of course, the choice of extension is not unique, and a good design is application dependent. The beamforming function can now be computed by the Fourier transform

$$
w(\boldsymbol{p})=\int_{\mathbb{R}^{n}} \hat{\omega}(\boldsymbol{r}) e^{-j 2 \pi\langle\boldsymbol{r}, \boldsymbol{p}\rangle} d \boldsymbol{r},
$$

which, for an arbitrary target filter, would be calculated numerically. However, the target and extended filter can be designed such that an exact analytical Fourier transform is available.

For example, when $\hat{w}(\boldsymbol{r})=\delta\left(\boldsymbol{r}-\boldsymbol{r}_{0}\right)$, where $\delta$ denotes the Dirac delta function, then $w(\boldsymbol{p})=e^{-j 2 \pi\left\langle\boldsymbol{r}_{0}, \boldsymbol{p}\right\rangle}$. This function, once sampled, corresponds to matched beamforming.

The $n$-dimensional ball indicator function,

$$
\hat{\omega}(\boldsymbol{r})= \begin{cases}1 & \text { if } \boldsymbol{r} \in \mathcal{B}\left(\boldsymbol{r}_{0}, R\right), \\ 0 & \text { otherwise }\end{cases}
$$

where $\boldsymbol{r}_{0} \in \mathbb{S}^{n-1}$ and $\mathcal{B}\left(\boldsymbol{r}_{0}, R\right)$ is the ball centered at $\boldsymbol{r}_{0}$ with radius $R>0$, also has an explicit Fourier transform:

$$
w(\boldsymbol{p})=R(2 \pi)^{n / 2}\|2 \pi p R\|^{-n / 2} J_{n / 2}(\|2 \pi p R\|) e^{-j 2 \pi\left\langle\boldsymbol{r}_{0}, \boldsymbol{p}\right\rangle},
$$

where $J_{n / 2}$ is the $n / 2$ th Bessel function of the first kind.

Finally, the $n$-dimensional symmetric Gaussian

$$
\hat{\omega}(\boldsymbol{r})=\frac{1}{(2 \pi)^{n / 2} \sigma^{n}} e^{-\frac{\left\|\boldsymbol{r}-\boldsymbol{r}_{0}\right\|^{2}}{2 \sigma^{2}}}
$$

with mean $\boldsymbol{r}_{0} \in \mathbb{S}^{n-1}$ and standard deviation $\sigma$, has Fourier transform

$$
w(\boldsymbol{p})=(2 \pi)^{n} e^{-2 \pi^{2} \sigma^{2}\|\boldsymbol{p}\|^{2}} e^{-j 2 \pi\left\langle\boldsymbol{p}, \boldsymbol{r}_{0}\right\rangle} .
$$

And of course, any linear combination of the above will also have an analytically tractable Fourier transform.

\subsection{Sampling to obtain weights}

Consider now $L$ actual antennas with positions $\boldsymbol{p}_{1}, \ldots, \boldsymbol{p}_{L} \in \mathbb{R}^{n}$. For antenna $i$ the beamforming weight $w_{i}$ is $\frac{w\left(\boldsymbol{p}_{i}\right)}{\beta}$, where $\beta=$ $\sqrt{\sum_{i=1}^{L}\left|w\left(\boldsymbol{p}_{i}\right)\right|^{2}}$ is a normalisation to avoid magnifying noise. How closely the beamforming achieves the target filter over $\mathbb{S}^{n}$ depends strongly on the number and position of the antennas. The resultant beam-shape can be shown to be

$$
b(\boldsymbol{r})=\frac{\hat{\omega}(\boldsymbol{r})}{\beta} *\left(\sum_{i=1}^{L} e^{-j 2 \pi\left\langle\boldsymbol{r}, \boldsymbol{p}_{i}\right\rangle}\right) \propto \hat{\omega}(\boldsymbol{r}) * b_{d}(\boldsymbol{r}),
$$

where $b_{d}(\boldsymbol{r})$ is the Point Spread Function (PSF) - also called dirty beam in radio astronomy. Hence, the ability of the beam-shape to approximate the target filter depends on the array layout. In particular, if the layout is sufficiently dense and isotropic, the PSF will be well-behaved (have fast decay), and the beam-shape will be a good approximation for the target filter. 


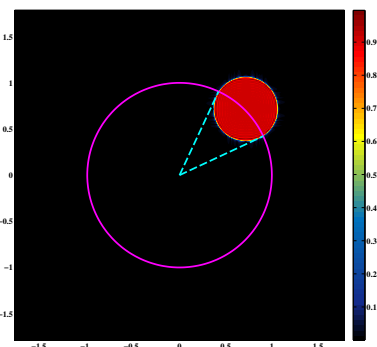

(a) Extended filter. Over the unit circle it filters signals with directions $25^{\circ}-65^{\circ}$.

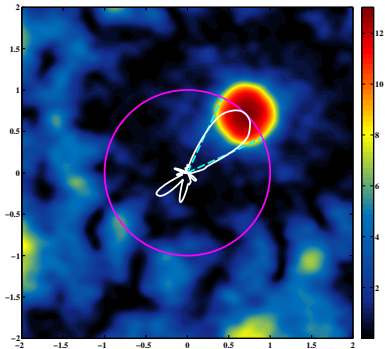

(c) Beam-shape obtained with beamforming weights (white line), which approximates relatively well the target.

Fig. 1: Filtering a range of directions with flexibeam for $\Theta=40^{\circ}$ and 96 antennas. The beam-shape covers a much wider range of directions than matched beamforming.

\subsection{Example using ball indicator function}

Working on the plane, suppose we were interested in signals with directions of arrival between $25^{\circ}$ and $65^{\circ}$. Hence, we get a target filter $\hat{w}(\theta)$ of the form

$$
\hat{w}(\theta)= \begin{cases}1 & \text { if } \theta \in\left[25^{\circ}, 65^{\circ}\right], \\ 0 & \text { otherwise, }\end{cases}
$$

where $\theta$ is an angle on the unit circle $\mathbb{S}^{1}$ measured in degrees. One extension $\hat{\omega}(\boldsymbol{r})$ to $\mathbb{R}^{2}$ is the ball indicator function $\left[5\right.$, with $\boldsymbol{r}_{0}=$ $(1,1) / \sqrt{2} \in \mathbb{S}^{1}, R=\sqrt{2} \sqrt{1-\cos \Theta}$ and $\Theta=40^{\circ}$ is the diameter angle of the target filter. Fig. 1a shows that over the unit circle it is equal to the target filter $w(\boldsymbol{r})$, while Fig. 1b shows the resultant beamforming function $w(\boldsymbol{p})$, and example beamforming weights determined by the array layout. The resultant beam-shape in Fig. 1c can be seen in general to be a good approximation. In contrast, matched beamforming would require steering towards many directions to cover the same area.

For a fixed number of antennas Fig.2a shows that for very small $\Theta$ the beam-shape is essentially identical to the one from matched beamforming, while for large $\Theta$, the beam-shape struggles to cover the whole range. For fixed $\Theta$ Fig. $2 \mathrm{~b}$ shows that the beam-shape becomes increasingly accurate as the number of antennas increases.

\section{WIFI TRANSMISSION AS EXAMPLE}

Consider the living room presented in Fig. 3 We would like to irradiate the WiFi signal optimally. The room is approximately $10.5 \mathrm{~m} \times$

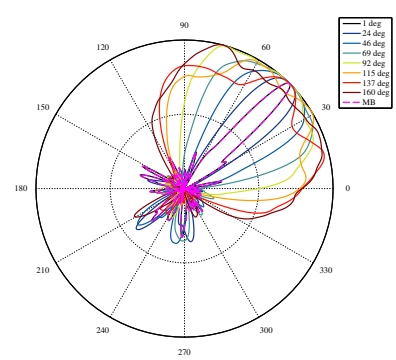

(a) Filtering a range of directions with flexibeam for various angles and 96 antennas.

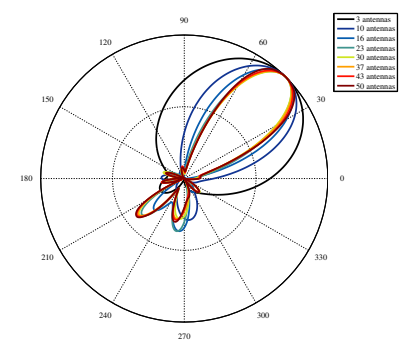

(b) Filtering a range of directions with flexibeam for $\Theta=40^{\circ}$ with varying number of antennas.
Fig. 2: Evolution of the flexibeam beam-shape for various angles and number of antennas.

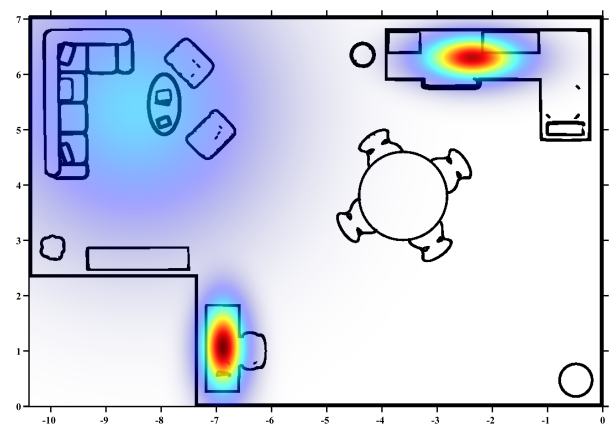

Fig. 3: Plan of the living room overlaid with the preference function, provided by the user. The router is in the bottom right corner.

$7 \mathrm{~m}$, and the WiFi router is located at the bottom right corner. To simplify, it and all other devices lie on a plane. The router has 27 antennas located at $\boldsymbol{p}_{1}, \ldots, \boldsymbol{p}_{27} \in \mathbb{R}^{2}$, and arranged on three concentric circles of radii 5,15 and $25 \mathrm{~cm}$ respectively. The router centre is designated by $\boldsymbol{p}_{0} \in \mathbb{R}^{2}$.

The inhabitant indicated by the coloured regions the locations where they are most likely to use their devices, and hence where good power coverage is needed. This defines what we call a preference function $f: \mathbb{R}^{2} \rightarrow \mathbb{R}$. The goal becomes then the design of an optimal beam-shape, which would cover the designated areas well while compensating for signal attenuation.

As is normally the case, every broadcasting device lies in the far field of all antennas. Moreover, WiFi signals can typically be considered narrow-band. Thus, the model in Section 2.1 is applicable, and the signal received by an antenna at position $\boldsymbol{p} \in \mathbb{R}^{2}$ can be written:

$$
x(t, \boldsymbol{p})=\int_{\mathbb{S}^{1}} s(t, \boldsymbol{r}) \stackrel{\gamma}{\gamma}(\boldsymbol{r}) e^{-j 2 \pi\langle\boldsymbol{r}, \boldsymbol{p}\rangle} d \Omega,
$$

where $s(t, \boldsymbol{r})$ is the summation of signals coming from direction $\boldsymbol{r}$, and $\stackrel{\gamma}{\gamma}(\boldsymbol{r})=\int_{\Gamma_{\boldsymbol{r}}} \frac{d \boldsymbol{l}}{\left\|\boldsymbol{l}-\boldsymbol{p}_{0}\right\|}$ the radial attenuation function.

Applying the beamforming function $\omega(\boldsymbol{p})$ is equivalent to applying a radial filter $\hat{w}(\boldsymbol{r})$ to the signals received by the antennas from each direction:

$$
y(t)=\int_{\mathbb{S}^{1}} s(t, \boldsymbol{r}) \stackrel{\circ}{\gamma}(\boldsymbol{r}) \hat{w}(\boldsymbol{r}) d \Omega
$$

with $\hat{w}(\boldsymbol{r}):=\iint_{\mathbb{R}^{2}} w(\boldsymbol{p}) e^{j 2 \pi\langle\boldsymbol{r}, \boldsymbol{p}\rangle} d \boldsymbol{p}$. To compensate for the attenuation and yield good coverage, $\hat{w}(\boldsymbol{r})=\frac{1}{\gamma(\boldsymbol{r})} \int_{\Gamma_{\boldsymbol{r}}} f(\boldsymbol{l}) d \boldsymbol{l}$ can 


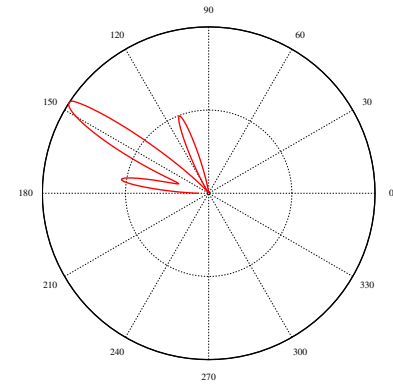

(a) Target filter.

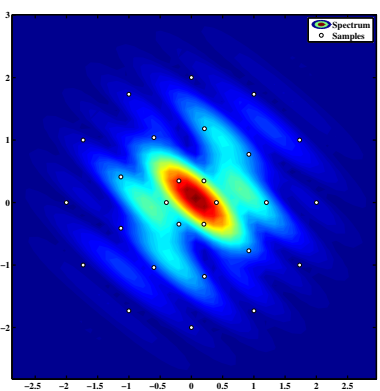

(c) The beamforming function. White dots show the locations to be sampled.

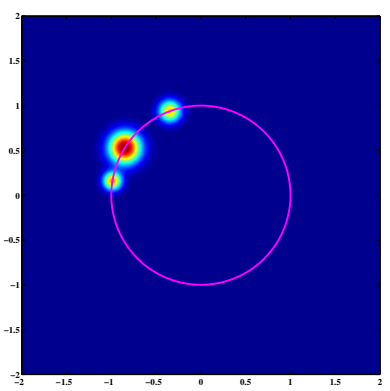

(b) Its $2 \mathrm{D}$ extension.

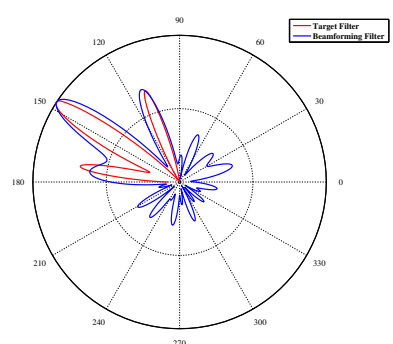

(d) The target filter is well approximated by the beam-shape.
Fig. 4: WiFi filter design and its performance.

be chosen. Thus, the desired filter is the radial projection of $f$ reweighted to compensate for attenuation. We can approximate $\hat{w}(\boldsymbol{r})$ on the circle by three Gaussians:

$$
\hat{w}(\theta) \simeq \sum_{i=1}^{3} \frac{\alpha_{i}}{\sqrt{2 \pi}} e^{-\frac{\left(\theta-\mu_{i}\right)^{2}}{2 \sigma_{i}^{2}}}
$$

where $\alpha_{i}, \mu_{i}, \sigma_{i}$ are respectively the weight, mean and standard deviation characterising each Gaussian. To compute the beamforming function $w(\boldsymbol{p})$, inspired by the approximate decomposition (7), we used

$$
\hat{\omega}(x, y)=\sum_{i=1}^{3} \frac{\alpha_{i}}{2 \pi \sigma_{i}} e^{-\frac{1}{2 \sigma_{i}^{2}}\left[\left(x-\cos \left(\mu_{i}\right)\right)^{2}+\left(y-\sin \left(\mu_{i}\right)\right)^{2}\right]},
$$

as plotted in Fig. 4b Then, $w(\boldsymbol{p})$, shown in Fig. 4c is the 2D Fourier transform of (8). The beam-shape of the router is then

$$
b(\boldsymbol{r})=\frac{1}{\beta} \sum_{i=1}^{27} w\left(\frac{\boldsymbol{p}_{i}}{\lambda}\right) e^{j 2 \pi\left\langle r, \frac{\boldsymbol{p}_{i}}{\lambda}\right\rangle},
$$

where $\beta=\sum_{i=1}^{27}\left|w\left(\frac{\boldsymbol{p}_{i}}{\lambda}\right)\right|^{2}$ is a normalisation factor. The radiation pattern, shown in Fig. 5 covers the regions of interest well. Given the finite number of antennas, the beam-shape approximates $\hat{w}(\boldsymbol{r})$, with unavoidable side lobes (Fig. 4d).

\section{CONCLUSIONS}

We observed that beamforming deployed today is primarily based on focusing on a single point. It seemed attractive to try to generalise

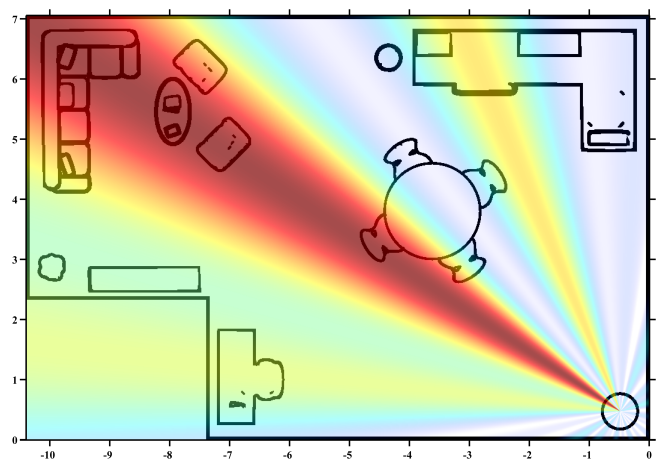

Fig. 5: Coverage achieved with flexibeam. The regions of interest are well covered by the router (sofa, desk and kitchen).

this in order to tackle problems that naturally want signal information from spatial regions, and to avoid the side-effects of trying to realise the most difficult filter of all: that of focusing on a single point.

To this end, we developed a new analytical technique called flexibeam that considers beamforming as a continuous function across space, and that beamforming weights are samples of this function.

The flexible design and tractable construction facilitates a broad range of applications. On a simple example, we showed that the target filter can be well approximated. Flexibeam takes advantage of the ever increasing number of antennas used in arrays. We believe it has great potential in applications varying from mobile phone networks, WiFi, ultrasound and radio astronomy. Future work will focus on their realisation.

\section{REFERENCES}

[1] D. H. Johnson and D. E. Dudgeon, Array signal processing: concepts and techniques. Simon \& Schuster, 1992.

[2] B. D. V. Veen and K. M. Buckley, "Beamforming: a versatile approach to spatial filtering," ASSP Magazine, IEEE, vol. 5, no. 2, pp. 4-24, 41988.

[3] M. S. Gast, 802.11 ac: A survival guide. O'Reilly Media, Inc., 2013.

[4] A. Sibille, C. Oestges, and A. Zanella, MIMO: from theory to implementation. Academic Press, 2010.

[5] L. W. Schmmer Jr, "Fundamentals of ultrasonic phased arrays," Modern Physics Letters B, vol. 22, no. 11, pp. 917-921, 2008.

[6] B. Rafaely, Fundamentals of Spherical Array Processing. Springer, 2015, vol. 8.

[7] J. F. Synnevag, A. Austeng, and S. Holm, "Adaptive beamforming applied to medical ultrasound imaging," Ultrasonics, Ferroelectrics, and Frequency Control, IEEE Transactions on, vol. 54, no. 8, pp. 1606-1613, 2007.

[8] O. Ocal, P. Hurley, G. Cherubini, and S. Kazemi, "Collaborative randomized beamforming for phased array radio interferometers," in Acoustics, Speech and Signal Processing (ICASSP), 2015 IEEE International Conference on, April 2015, pp. 56545658. 